\title{
Valence and spectral properties of rare-earth clusters
}

\author{
L. Peters,,${ }^{1, *}$ I. Di Marco, ${ }^{2}$ M. S. Litsarev,${ }^{3}$ A. Delin, ${ }^{2,4}$ M. I. Katsnelson, ${ }^{1}$ A. Kirilyuk, ${ }^{1}$ B. Johansson,,${ }^{2,5}$ \\ B. Sanyal, ${ }^{2}$ and O. Eriksson ${ }^{2}$ \\ ${ }^{1}$ Radboud University Nijmegen, Institute for Molecules and Materials, NL-6525 AJ Nijmegen, The Netherlands \\ ${ }^{2}$ Department of Physics and Astronomy, Uppsala University, Box 516, SE-75120, Uppsala, Sweden \\ ${ }^{3}$ Skolkovo Institute of Science and Technology, Novaya St. 100, Skolkovo, Odintsovsky District, 143025 Moscow Region, Russia \\ ${ }^{4}$ Department of Nano and Materials Physics, Royal Institute of Technology (KTH), Electrum 229, SE-16440 Kista, Sweden \\ ${ }^{5}$ Department of Materials Science and Engineering, Royal Institute of Technology (KTH), SE 10044 Stockholm, Sweden
}

(Received 19 February 2015; revised manuscript received 3 July 2015; published 24 July 2015)

\begin{abstract}
The rare earths are known to have intriguing changes of the valence, depending on the chemical surrounding or geometry. Here, we aim at predicting the transition of valence when passing from the atomic divalent limit to the bulk trivalent limit. This transition is analyzed by addressing clusters of various size for selected rare-earth elements, i.e., $\mathrm{Sm}, \mathrm{Tb}$, and $\mathrm{Tm}$, via a theoretical treatment that combines density functional theory with atomic multiplet theory. Our results show that Tm clusters change from pure divalent to pure trivalent at a size of six atoms, while $\mathrm{Tb}$ clusters are already divalent for two atoms and stay so until eight atoms and the bulk limit. Instead, Sm clusters are respectively purely divalent up to eight atoms. For larger Sm clusters, a transition to a trivalent configuration is expected and likely accompanied by a regime of mixed valence. The valence of all rare-earth clusters, as a function of size, is predicted from the interpolation of our calculated results. These predictions are argued to be best investigated by spectroscopic measurements. To ease experimental analysis, we provide theoretical spectra, based on dynamical mean-field theory in the Hubbard I approximation.
\end{abstract}

DOI: 10.1103/PhysRevB.92.035143

PACS number(s): 31.15.A-, 36.40.-c, 75.30.Mb, 79.60.Jv

\section{INTRODUCTION}

Recently, the total magnetic and dipole moments of isolated rare-earth clusters ( $\mathrm{Pr}, \mathrm{Tb}, \mathrm{Ho}$, and $\mathrm{Tm}$ ) in the gas phase have been measured experimentally for a size range of 5-30 atoms [1,2]. These experiments show a very interesting and unexpected behavior completely different from the bulk. For example, for $\mathrm{Tb}$ clusters, the magnetic moment oscillates heavily as a function of cluster size, while for Tm and Pr clusters there is almost no size dependence. Further, there appears to be a large electric dipole moment for certain Tm cluster sizes. Understanding the principles behind this behavior is important not only from a fundamental point of view, but also for possible applications at the nanoscale.

However, before magnetism can be addressed, an absolutely crucial information is necessary. In fact, all physical properties of rare-earth clusters require the knowledge of the number of $4 f$ electrons, or equivalently of $s p d$ electrons. Determining these numbers is often referred to as determining the valence of the cluster. Magnetism is the most significant example of the importance of determining the correct valence of rare-earth clusters. In fact, the $4 f$ electrons constitute the local magnetic moments while the $s p d$ electrons mediate the coupling between them. It is evident that changing the valence, i.e., promoting an electron from the $s p d$ states to the $4 f$ states, or vice versa, may lead to very different magnetic properties. Although determining the valence is of a crucial importance, it is also difficult to tackle this problem from a technical point of view. Further, it is complicated by the fact that the valence of the rare earths is known from previous works to depend delicately on the chemical surrounding or geometry. For instance, it is known that the surface of elemental Sm

\footnotetext{
*1.peters@science.ru.nl
}

is divalent [3], whereas the bulk is trivalent. It is now well established that with the exception of $\mathrm{Eu}, \mathrm{Yb}$ and the $\alpha$ phase of $\mathrm{Ce}$, all rare-earth elements form trivalent configurations in the solid [4]. Eu and $\mathrm{Yb}$ are divalent, because this configuration provides a half-filled or filled $4 f$ shell [4]. On the contrary, the isolated rare-earth atoms are all divalent with the exception of $\mathrm{La}, \mathrm{Ce}, \mathrm{Gd}$, and $\mathrm{Lu}$, which are trivalent. The transition from atom to solid can be seen as a function of clusters with increasing size where the valence of the end points is known precisely. The valence of clusters is completely unknown and it is reasonable to ask how the transition from divalent to trivalent occurs as a function of increasing cluster size, for what size of clusters it happens and if mixed valence configurations are possible. Answering these precise questions is the main aim of the present study.

Choosing a theoretical method that is adequate for investigating the cluster regime is not a simple task. It is well known that density functional theory (DFT) [5,6] in local density approximation (LDA) or generalized gradient approximation (GGA) is inadequate [7-10] for rare-earth bulk systems. This failure is caused by the localized $4 f$ electrons, for which the electron-electron repulsion is strong and cannot be described properly by functionals derived in the limit of a nearly uniform electron gas. Although, in principle, quantum chemistry methods [11] such as configuration interaction (CI) offer an adequate treatment of strong correlations, the prohibitive computational cost for treating $f$ electrons makes this approach feasible only for the dimers of the early rare-earth elements, in particular, if optimization of atomic positions is addressed. In order to have a systematic view of clusters of different size across the rare-earth series, we decided instead to exploit the fact that the $4 f$ electrons hybridize little with the rest. Then we can evaluate the electronic structure, total energies, and valence of selected rare-earth clusters by using DFT with $4 f$ electrons treated as core states in combination 
with the Born-Haber cycle [12,13]. The spectral properties are instead obtained by the DFT+DMFT [14] approach in the limit of zero hybridization, i.e., in the Hubbard I approximation (HIA) [15]. Notice that, although the validity of DMFT is usually assessed with respect to the number of nearest neighbors, this theory is also exact in the limit of zero hybridization [14]. Before a HIA calculation can be performed, the geometry, the valence, the Hubbard $U$ parameter, and the position of the first $4 f$ peak below the Fermi level should be determined. Once this has been done, then the HIA can be used to determine the spectral properties of the rare-earth cluster. For the geometry calculations, the $4 f$ electrons are made chemically inert by treating them as part of the core [16]. The valence and position of first $4 f$ peak below the Fermi level are calculated by following the Born-Haber cycle as presented in Refs. [12,13]. Our calculations are focused on three selected elements, i.e., Sm, Tb, and Tm, since Sm and Tm are known to have small energy differences between a trivalent and divalent configuration in the bulk [12], and due to recent experimental interest in these three elements $[1,2]$. Results for those elements are then interpolated to obtain information over the whole series of rare-earth clusters.

\section{THEORY}

\section{A. Valence}

Determining the valence requires to calculate the total energy difference between divalent, $f^{n+1}[s p d]^{2}$, trivalent, $f^{n}[s p d]^{3}$ and mixed valence configurations. Unfortunately, evaluating those energies directly in conventional DFT (LDA or GGA) is a hopeless task, due to failure in treating strong correlations among the $4 f$ electrons [12,17]. For localized systems, where the strong Coulomb repulsion is dominant with respect to the hybridization, one can resort to the Born-Haber cycle. The idea behind this approach is to exploit the fact that the $4 f$ shell is so localized that it is essentially the same in the atom as in the isovalent cluster. This crucial assumption makes it possible to devise a computational scheme combining atomic experimental information with simplified DFT calculations, where both intra and inter $4 f$ couplings are neglected.

The Born-Haber cycle is schematically depicted in Fig. 1. The aim of the cycle is to calculate the energy difference $E$ between a purely divalent cluster and a purely trivalent cluster. As mentioned above, $E$ cannot be accurately evaluated by direct DFT calculations, due to the lack of a proper description of the localized $4 f$ shell. Nevertheless, one can evaluate the energies $E(\text { II })_{\text {cluster }}$ and $E(\text { III })_{\text {cluster }}$ for clusters where the $4 f$ electrons are treated as chemically inert (see next section). From these energies, one can recover the correct cluster energies by adding the contributions due the inter and intra $4 f$ couplings. To a good approximation, the difference between the inter $4 f$ couplings, $E_{C, f \rightarrow[s p d]}(\mathrm{III})_{\text {cluster }}$ and $E_{C, f \rightarrow[s p d]}(\mathrm{II})_{\text {cluster }}$, can be neglected $[12,17]$. Thus it remains to compute the energy difference between the intra $4 f$ couplings, $E_{C, f \rightarrow f}(\mathrm{III})_{\text {cluster }}$ and $E_{C, f \rightarrow f}(\mathrm{II})_{\text {cluster. }}$. This difference is still not accessible with sufficient accuracy in DFT based methods. This problem can be circumvented by going around the Born-Haber cycle via the atomic energies, since these intra $4 f$ couplings then cancel with their isovalent
TRIVALENT

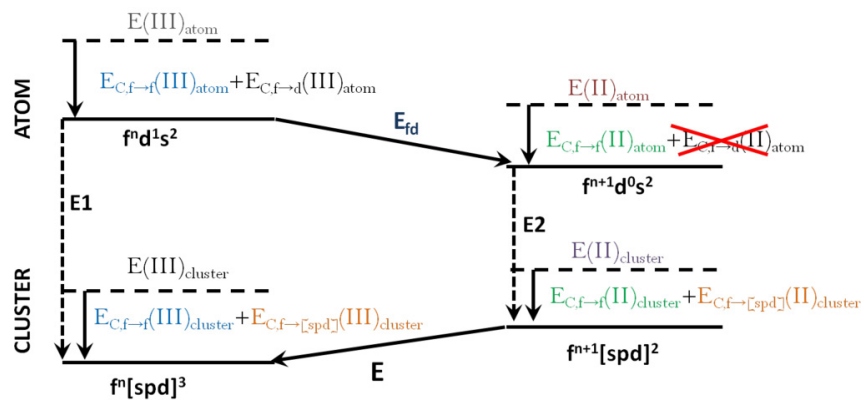

FIG. 1. (Color online) Schematic picture of the Born-Haber cycle [12]. The dotted lines represent total energies without intra and inter $4 f$ coupling, while the full lines corresponds to full total energy. Further, $E_{f d}$ is the atomic $f$ to $d$ promotion energy, $E_{C, f \rightarrow d}(\mathrm{II})_{\text {atom }}$ and $E_{C, f \rightarrow d}(\mathrm{III})_{\text {atom }}$ refer to the coupling between the $4 f$ and $5 d$ shell of, respectively, the divalent and trivalent atoms, $E_{C, f \rightarrow[s p d]}(\mathrm{III})_{\text {cluster }}$ and $E_{C, f \rightarrow[s p d]}(\mathrm{III})_{\text {cluster }}$ correspond to the coupling between the $4 f$ shell and $s p d$ states of, respectively, the divalent and trivalent clusters, and $E_{C, f \rightarrow f}$ terms refer to intra $4 f$ shell couplings.

atomic counterparts. However, by going around the cycle in this way, three new quantities have to be introduced, i.e., $E_{f d}$, $E_{C, f \rightarrow d}(\mathrm{III})_{\text {atom }}$, and $E_{C, f \rightarrow d}(\mathrm{II})_{\text {atom. }}$. The former is the energy required to promote a $4 f$ electron to the $5 d$ shell in the atom and the latter two represent the coupling energy between the $4 f$ shell and the $5 d$ shell. Since there is no $5 d$ electron in the divalent atom, $E_{C, f \rightarrow d}(\mathrm{II})_{\text {atom }}$ is zero, while the other two are known experimentally $[12,18,19]$. There is now one last step needed to complete the Born-Haber cycle and obtain the energy difference $E$. The energy difference between the decoupled (intra and inter $4 f$ coupling neglected) isovalent atom and cluster must be accurately computed. Luckily, this energy difference, which is often referred to as generalized cohesive energy, is known to be reproduced very well by DFT with a chemically inert $4 f$ shell $[12,17]$.

The concepts outlined above can be easily extended to more general configurations, e.g., mixed valence configurations. The expression for the energy difference per atom $E$ between a pure trivalent and mixed valence configuration thus becomes

$$
\begin{aligned}
E= & {\left[E(\mathrm{III})_{\text {atom }}-E(\mathrm{III})_{\text {cluster }}\right] } \\
& -\left[\frac{n_{\text {div }}}{n_{\text {tot }}} E(\mathrm{II})_{\text {atom }}+\frac{n_{\text {triv }}}{n_{\text {tot }}} E(\mathrm{III})_{\text {atom }}-E(\text { mix })_{\text {cluster }}\right] \\
& -\frac{n_{\text {div }}}{n_{\text {tot }}}\left[E_{f d}+E_{C, f \rightarrow d}(\mathrm{III})_{\text {atom }}\right] .
\end{aligned}
$$

Here, $n_{\text {tot }}$ is the total number of atoms in the pure trivalent and mixed valence cluster, and $n_{\text {div }}$ and $n_{\text {triv }}$ correspond, respectively, to the number of divalent and trivalent atoms in the mixed valence cluster. Further, $E$ (III) $)_{\text {cluster }}$ and $E(\operatorname{mix})_{\text {cluster }}$ correspond to the total energies per atom of trivalent and mixed valence clusters where both intra and inter $4 f$ coupling has been neglected. Similarly, $E(\text { III) })_{\text {atom }}$ and $E(\mathrm{II})_{\text {atom }}$ are the total energies of trivalent and divalent atoms where the intra and inter $4 f$ couplings have been neglected. Finally, $E_{f d}$ and $E_{C, f \rightarrow d}(\mathrm{III})_{\text {atom }}$ are the atomic correction energies that are obtained from experiment. Note that the first term enclosed in square brackets in Eq. (1) is the generalized cohesive energy of a trivalent configuration. 
Furthermore, when $E(\mathrm{mix})_{\text {cluster }}$ corresponds to a purely divalent configuration $\left[n_{\text {div }}=n_{\text {tot }}\right.$ and $n_{\text {triv }}=0$ in Eq. (1)], then the second term enclosed in square brackets in Eq. (1) refers to the divalent generalized cohesive energy.

\section{B. First $4 f$ peak position}

The calculation of the first $4 f$ peak below the Fermi level can be made by following the procedure outlined in Ref. [13]. In this work the first $4 f$ peak below the Fermi level is calculated for all elemental bulk rare-earth systems. The first thing to consider is that the time scale on which a photoemission process takes place is too short for the geometry to adjust to the missing electron. Second, let us consider a bulk system where a $4 f$ electron is removed from one of the lattice sites, which is consistent with the fact that the $4 f$ electrons are localized. This process can be artificially divided into two steps: (1) a $4 f$ electron is promoted to the valence band and (2) an electron is adiabatically taken out of the valence band. In the bulk, the ionization energies of the ground state and the excited state with one $4 f$ electron promoted to the valence band are virtually the same, due to that the $4 f$ hole is efficiently screened by the valence electrons. This means that with respect to this ionization energy the first $4 f$ peak position corresponds to the total energy difference between the ground state and the state in which one atom is replaced with one $4 f$ electron less and one valence electron more. Thus again the Born-Haber cycle can be exploited here.

Extending the previous procedure to cluster is not straightforward, as it is not clear whether the valence electrons are able to fully screen the $4 f$ hole or not. In the case of bulk systems, there is an infinite number of sites which can provide valence electrons to screen the $4 f$ hole created at one atomic site. However, in case of our clusters, there are less than nine sites in total, which means that we cannot neglect the formation of the $4 f$ hole at one atomic site. The photoemission process may in fact lead to a difference in ionization energy between the ground state and the excited state with one $4 f$ electron promoted to the valence states. This difference can be estimated with a good accuracy by the difference in the eigenvalues of the highest occupied Kohn-Sham orbital of these states.

\section{DETAILS OF CALCULATIONS}

All the calculations in this report are performed with a full potential linear muffin-tin orbital (FP-LMTO) method [20]. A GGA parametrization of the exchange-correlation functional as formulated by Perdew, Burke, and Ernzerhof is used [21]. Since the used FP-LMTO program is originally designed for periodic bulk systems, a large cubic unit cell of 16 - $\AA$ dimension is used to prevent the interaction between clusters of different unit cells. Furthermore, the calculations are performed by considering only the gamma point. The basic geometrical setup and the details of the basis set are the same for all calculations, as explained in the following. A muffin-tin radius of 2.6 a.u. is used for all atoms. The main valence basis functions are chosen as $6 s, 6 p$, and $5 d$ states, while $5 s$ and $5 p$ electrons are treated as pseudocore in a second energy set [20]. The $4 f$ states are treated as valence states for the Hubbard I calculations, while for all the other calculations they are treated as core states. In the latter case, $5 f$ states are added to the valence electrons. Including some states with $f$ angular character is important to increase the precision of the basis set and obtain accurate total energies. Three kinetic energy tails are used for $6 s$ and $6 p$ states [20], with values $-0.3,-2.8$, and -1.6 Ry. Further, a Hubbard $U$ of $8 \mathrm{eV}$ is used for the Hubbard I calculations of the spectral properties of the Sm, Tb and Tm clusters. This value of $U$ is commonly used for the $4 f$ shell of the rare earths [10,22-24]. In addition to the Hubbard $U$ parameter also the onsite exchange interaction $J$ is needed. For the $J$, it is well known that it is almost system independent $[25,26]$ and is therefore taken to be $1 \mathrm{eV}$ [22-24].

The many-body problem defined by the Hubbard $U$ and the onsite exchange $J$ can now be solved in the HIA. The self-energy function, which accounts for the strong correlation effects, is obtained by solving the atomiclike problem associated to the following Hamiltonian:

$$
\hat{H}_{\boldsymbol{R}}^{\mathrm{at}}=\hat{H}_{\boldsymbol{R}}^{\mathrm{DFT}}+\hat{H}_{U}-\mu_{\mathrm{at}} \sum_{\xi} \hat{c}_{\boldsymbol{R} \xi}^{\dagger} \hat{c}_{\boldsymbol{R} \xi} .
$$

Here, $\hat{c}_{\boldsymbol{R} \xi}^{\dagger}$ and $\hat{c}_{\boldsymbol{R} \xi}$ correspond to the creation and annihilation operators for a set of local orbitals $|\boldsymbol{R}, \xi\rangle$, where $\xi$ represents atomic like quantum numbers. The first term $\hat{H}_{R}^{\mathrm{DFT}}$ contains the DFT single-particle Hamiltonian projected onto the strongly correlated states. In our case, this Hamiltonian contains also the contribution of the spin-orbit coupling. The second term instead represents the effects due to on-site Coulomb repulsion between the strongly correlated orbitals. Finally, the last term is due to the real chemical potential of the atomic system and to the double counting of the Coulomb repulsion term, as described in Ref. [22]. These two contributions combine into an effective chemical potential $\mu_{\mathrm{at}}$, which is then used to embed the atom in the cluster. In general, there are several possible choices for the double counting correction [14] from the correlated problem itself but for clusters it is more advantageous to use the information obtained through the Born-Haber cycle. We notice in fact that the last term in Eq. (2) is unambiguously determined by the number of $4 f$ electrons and by the position of the first $4 f$ peak below the Fermi level. These quantities are calculated from the Born-Haber cycle in our computational scheme, and are reported in Figs. 2 and 3, respectively. More details on the implementation of the Hubbard I routine that is used in this work are given elsewhere [22,27-30], and we refer the reader to those studies for a complete description of our methods.

Finally, for the calculation of the valence, different starting geometries have been considered and optimized for each valence configuration separately. The optimization was perfomed by means of the wrapped polyhedron method, which offers excellent convergence to the minimal energy, even in presence of several local minima [31]. For the other calculations, i.e., the first $4 f$ peak position below the Fermi level and $4 f$ PDOS, the geometry is fixed to the thus found lowest-energy geometry.

\section{RESULTS}

\section{A. Valence}

By applying the Born-Haber cycle to $\mathrm{Sm}, \mathrm{Tb}$, and $\mathrm{Tm}$ clusters in a size range of 2-8 atoms we obtain the valence, 


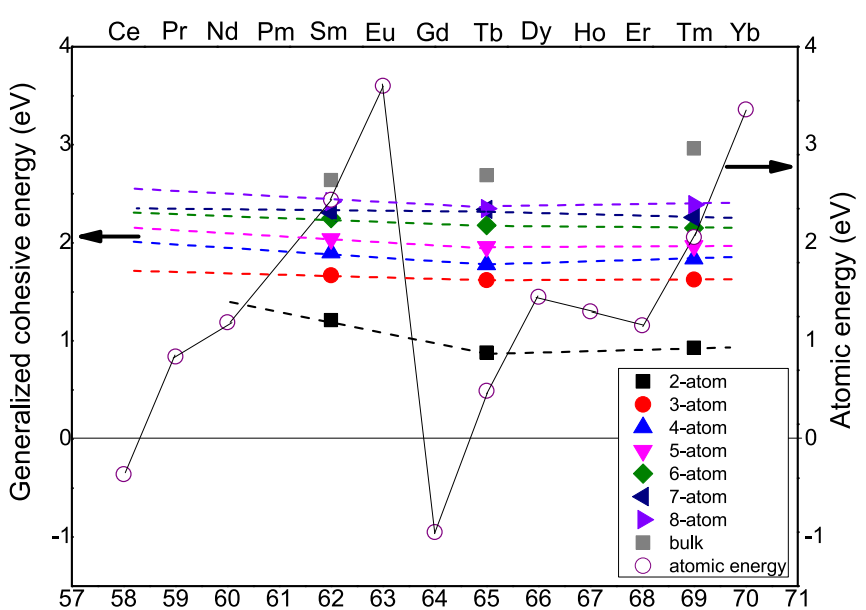

FIG. 2. (Color online) Results of valence calculation for $\mathrm{Sm}, \mathrm{Tb}$, and Tm clusters from $2-8$ atoms. On the left vertical axis, the difference between a pure divalent and trivalent generalized cohesive energy (in eV) is printed for the different cluster sizes. The open circles connected by the solid line correspond to the atomic correction energy (in eV) [12], shown on the right vertical axis. The dashed lines represent the simple linear extrapolations between the $\mathrm{Sm}, \mathrm{Tb}$, and $\mathrm{Tm}$ data points. The points where the solid line of the atomic correction energy crosses the dashed line correspond to the valence transitions. In the cases where the dashed line is below the solid line, the system is divalent and in the opposite cases it is trivalent. Finally, the grey squares correspond to the bulk generalized cohesive energies.

as illustrated in Fig. 2. In this graph, we show the difference in the generalized cohesive energy between a pure trivalent and divalent configuration for different sized clusters. We also show the atomic correction energies $\left(E_{f d}\right.$ plus $E_{C, f \rightarrow d}$ as described in Fig. 1). From Eq. (1), it is clear that a trivalent state is more favorable when the generalized cohesive energy difference is larger than the sum of atomic correction energies. The data reported in Fig. 2 illustrate that the generalized cohesive energy increases with cluster size, which is due to that more chemical bonds can be formed. Hence one may expect in general that as the clusters become larger, the divalent state of the atom should become less favorable. This is indeed what happens, as shown in Fig. 2. After having discussed the general trends, we can now focus on the selected elements. $\mathrm{Sm}$ is divalent for all clusters investigated in this study, which means that a transition from divalent to trivalent should occur for cluster sizes just above 8. Conversely, $\mathrm{Tb}$ is trivalent for all clusters analyzed in this study, as well as in the bulk form. This means that the transition from divalent to trivalent happens already for the cluster of smallest size, i.e., the dimer. Finally, Tm exhibits a more interesting behavior, since there is a change from divalent to trivalent at a size of six atoms. For increasing cluster size, all the three elements $\mathrm{Sm}, \mathrm{Tb}$, and Tm slowly approach their bulk generalized cohesive energies values of respectively $2.64,2.69$, and $2.96 \mathrm{eV}$ [17]. It has been pointed out that these bulk generalized cohesive energies change only gradually through the whole rare-earth series [18]. Extending this trend to the cluster regimes, the calculated points for $\mathrm{Sm}, \mathrm{Tb}$, and $\mathrm{Tm}$ can be extrapolated to make predictions for all rare-earth clusters up to a size of eight atoms. These extrapolated data are represented as dashed lines in Fig. 2.

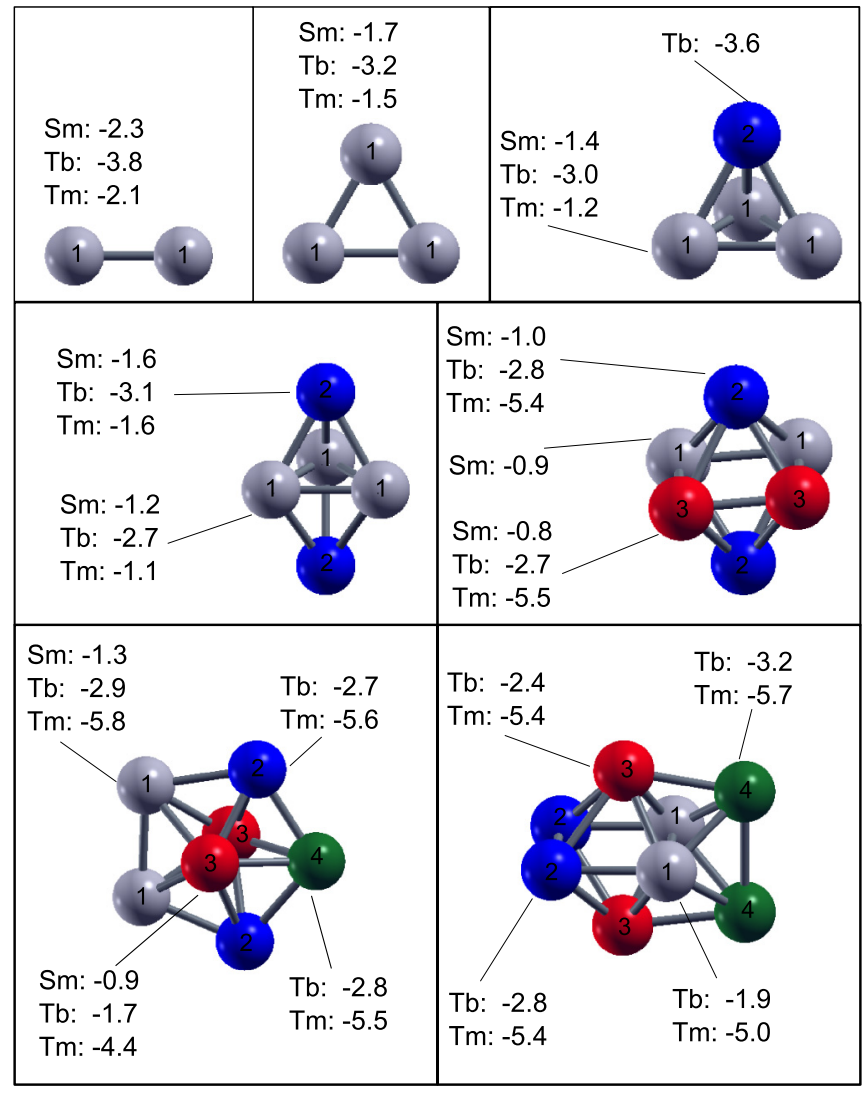

FIG. 3. (Color online) Results of the calculated site-dependent first $4 f$ peak position below the Fermi level for $\mathrm{Sm}, \mathrm{Tb}$, and $\mathrm{Tm}$ clusters from 2-8 atoms. Equivalent atoms are depicted with the same color and labeled with the same number. The value following the rare-earth element corresponds to the first $4 f$ peak position below the Fermi level in $\mathrm{eV}$ for that atomic type. For clusters of four and six atoms, respectively, the atom with number 2 and the atoms with number 1 are only inequivalent for the elements indicated. Thus, for $\mathrm{Tb}_{6}$ and $\mathrm{Tm}_{6}$, sites 1 and 3 are equivalent, and for $\mathrm{Sm}_{7}$ sites, 1, 2, and 4 are equivalent.

These general data can now be used for further analysis. For example, the dimers of Dy, Ho and Er are predicted to be divalent, while clusters of larger size should become trivalent. Note, however, that the behavior of the generalized cohesive energy of the Sm dimer somewhat differs from the rest, i.e., 3-8 atom clusters and the bulk. This discrepancy is the result of a larger $5 d$ electron contribution to the binding for the Sm dimer compared to the $\mathrm{Tb}$ and $\mathrm{Tm}$ dimer. For clusters larger than two atoms this difference in binding becomes negligible small. Thus the extrapolation for the dimers should be interpreted with a little bit of caution.

So far, only pure divalent and trivalent configurations have been compared. However, Eq. (1) is also used to consider possible mixed valence configurations. As an example the results for $\mathrm{Tb}_{3}, \mathrm{Tm}_{5}, \mathrm{Tm}_{6}, \mathrm{Sm}_{7}$, and $\mathrm{Sm}_{8}$ are shown in Table I. Here, the latter four are chosen, because they are close to a valence transition. Contrary, $\mathrm{Tb}_{3}$ is a simple example that is far from a valence transition. Note that all (mixed) valence configurations are compared with respect to the pure trivalent configuration. Thus a positive energy difference in the last 
TABLE I. The energy difference between the pure trivalent $E_{\text {triv }}$ and other possible configurations $E_{\mathrm{x}}$ is given in column three in $\mathrm{eV}$ per atom. Here, a positive number means that the pure trivalent configuration is more favorable and negative values mean that some or all atoms will be in the divalent configuration. In the second column, the first and second number represent, respectively, the total number of divalent atoms $n_{\text {div }}$ and trivalent atoms $n_{\text {triv }}$ of configuration $x$. The first column describes the system.

\begin{tabular}{|c|c|c|}
\hline System & $\begin{array}{l}\text { Configuration } \mathrm{x} \\
\quad\left(n_{\text {div }}-n_{\text {triv }}\right)\end{array}$ & $\begin{array}{l}E_{\text {triv }}-E_{\mathrm{x}} \\
(\mathrm{eV} / \text { atom })\end{array}$ \\
\hline \multirow[t]{3}{*}{$\mathrm{Tb}_{3}$} & $3-0$ & 1.1 \\
\hline & $2-1$ & 0.99 \\
\hline & $1-2$ & 0.57 \\
\hline \multirow[t]{5}{*}{$\mathrm{Tm}_{5}$} & $5-0$ & -0.1 \\
\hline & $4-1$ & 0.06 \\
\hline & $3-2$ & 0.06 \\
\hline & $2-3$ & 0.03 \\
\hline & $1-4$ & 0.02 \\
\hline \multirow[t]{6}{*}{$\operatorname{Tm}_{6}$} & $6-0$ & 0.09 \\
\hline & $5-1$ & 0.19 \\
\hline & $4-2$ & 0.16 \\
\hline & $3-3$ & 0.17 \\
\hline & $2-4$ & 0.14 \\
\hline & $1-5$ & 0.07 \\
\hline \multirow[t]{7}{*}{$\mathrm{Sm}_{7}$} & $7-0$ & -0.13 \\
\hline & $6-1$ & 0.12 \\
\hline & $5-2$ & 0.16 \\
\hline & $4-3$ & 0.08 \\
\hline & $3-4$ & 0.05 \\
\hline & $2-5$ & 0.11 \\
\hline & $1-6$ & 0.16 \\
\hline \multirow[t]{8}{*}{$\mathrm{Sm}_{8}$} & $8-0$ & -0.02 \\
\hline & $7-1$ & 0.07 \\
\hline & $6-2$ & 0.14 \\
\hline & $5-3$ & 0.09 \\
\hline & $4-4$ & 0.24 \\
\hline & $3-5$ & -0.01 \\
\hline & $2-6$ & 0.03 \\
\hline & $1-7$ & 0.06 \\
\hline
\end{tabular}

column of Table I means that that configuration is less stable than the pure trivalent configuration. In case of a negative value, instead, that configuration is predicted to be more stable than the trivalent one, i.e., the ground state of the system will have some or all atoms in the divalent configuration.

Table I shows that for Tm there is an abrupt change from pure divalent to trivalent, when the cluster size changes from 5 to 6 . These pure states are favorable over mixed valence states by roughly less than $0.1 \mathrm{eV} /$ atom. This abrupt valence change appears rather unexpectedly, having in mind that rough surfaces of Tm bulk are divalent [32]. For $\mathrm{Sm}_{7}$ and $\mathrm{Sm}_{8}$, also the pure divalent state is preferred over the mixed valence states by about 0.1 and $0.01 \mathrm{eV} /$ atom respectively. We can estimate $[12,13,17,18]$ the errors involved in our calculations to be of the order of $0.1 \mathrm{eV} /$ atom, which makes it difficult to resolve energy differences of this size. Moreover, at finite temperatures, the mixed valence configurations could become more favorable due to their higher entropy. Thus the absence of mixed valence configurations in Table I, as justified by energy differences of $0.1 \mathrm{eV} /$ atom or smaller, cannot be rigorously used to exclude mixed valence states of these clusters. In experiments on rare-earth clusters incorporated in an $\mathrm{Ar}$ matrix, abrupt valence changes were indeed observed [33,34]. In Ref. [34], the valence transition for Sm and Tm clusters is observed at a size of respectively six and ten atoms, which is in agreement with our results. However, the results of Ref. [33] for Pr, Nd, and Sm clusters do not agree with our data. This is likely due to the fact that in the experiments of Refs. [33,34] it is very hard to accurately estimate the cluster size.

\section{B. Spectral properties}

We now turn our attention to the calculation of the spectral properties, since spectroscopy is the most natural way to experimentally detect the valence and electronic properties in general. As discussed above, we are going to evaluate spectral functions in the HIA. Before the HIA can be used, the effective chemical potential in Eq. (2) can be calculated without ad hoc assumptions if the number of $4 f$ electrons and the position of the first $4 f$ peak below the Fermi level are known. The latter can be calculated as described in Sec. III, and the results of these calculations are reported in Fig. 3. Note, some atoms in the clusters may be equivalent, which reduces significantly the degrees of freedom of the needed calculations. In Fig. 3. equivalent atoms are represented by spheres of the same color and labeled with the same number. For example, in the cluster with five atoms there are only two atomic types to calculate, i.e., the grey atoms " 1 " and the blue atoms "2." However, clusters of the same size but of different elements may have different symmetry. For example, the cluster with four atoms has two inequivalent atomic types for $\mathrm{Tb}$ but only 1 for $\mathrm{Sm}$ and Tm. In this case, the figure is drawn for the lowest possible symmetry, and a higher symmetry has to be intended if numeric values are not given. In other words, for the skipped rare-earth elements these are equivalent atoms. Finally, notice that for the eight-atom cluster information on $\mathrm{Sm}$ is not included, because it has a completely different geometry.

The calculations of the first $4 f$ peak position below the Fermi level presented in Fig. 3 are for the ground state. For $\mathrm{Sm}$ and $\mathrm{Tb}$, this means, respectively, a divalent and trivalent configurations up to a cluster size of 8 . It can be observed that this peak position is predicted to be quite site dependent. Also, for Tm, the binding energy of the first $4 f$ peak below the Fermi level decreases, when the valence transition (at a cluster size of 6 atoms) is approached. This trend is also clear for $\mathrm{Sm}$ clusters, as Fig. 3 shows. Hence, data in this figure, as well as the data in Fig. 2, suggest that also for Sm a valence transition will occur, for a cluster size larger than 8 atoms. Interestingly, divalent $\mathrm{Sm}$ is nonmagnetic, while trivalent $\mathrm{Sm}$ is magnetic according to Hund's rules. For Tb, it is clear that the first $4 f$ peak below the Fermi level is approaching the bulk position, which is at $2.2 \mathrm{eV}$ below the Fermi level [13]. Similarly the first $4 f$ peak positions below the Fermi level of $\mathrm{Tm}_{6}-\mathrm{Tm}_{8}$ are already quite close to that of trivalent Tm bulk (at $4.5 \mathrm{eV}$ below the Fermi level [13]).

Finally, with the valence (Fig. 2) and first $4 f$ peak position (Fig. 3) at hand, HIA calculations are performed without ad hoc parameters. More precisely, the $4 f$ partial density of states (4f PDOS) for $\mathrm{Tb}_{3}, \mathrm{Tm}_{5}$, and $\mathrm{Tm}_{6}$, and $\mathrm{Sm}_{7}$ are calculated in 


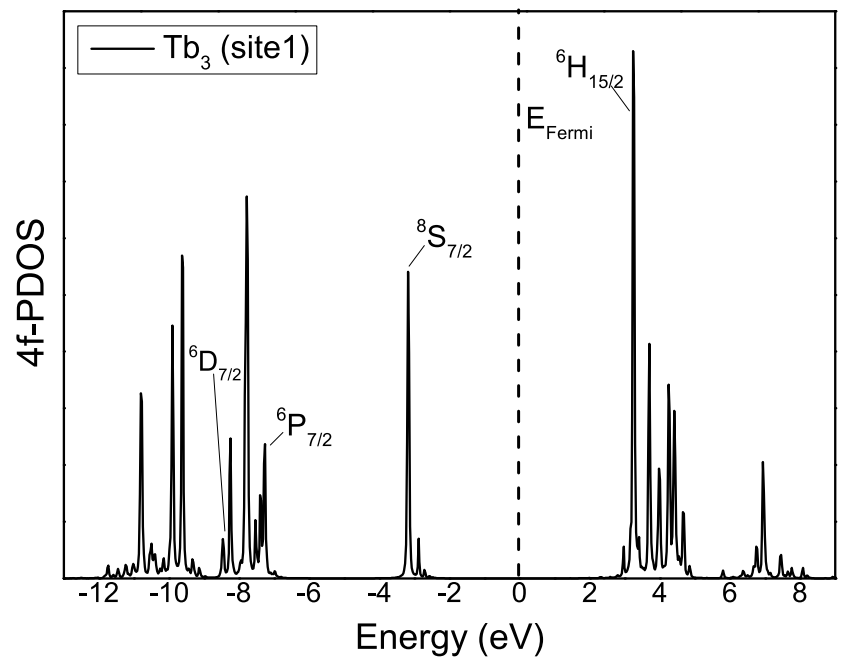

(a)

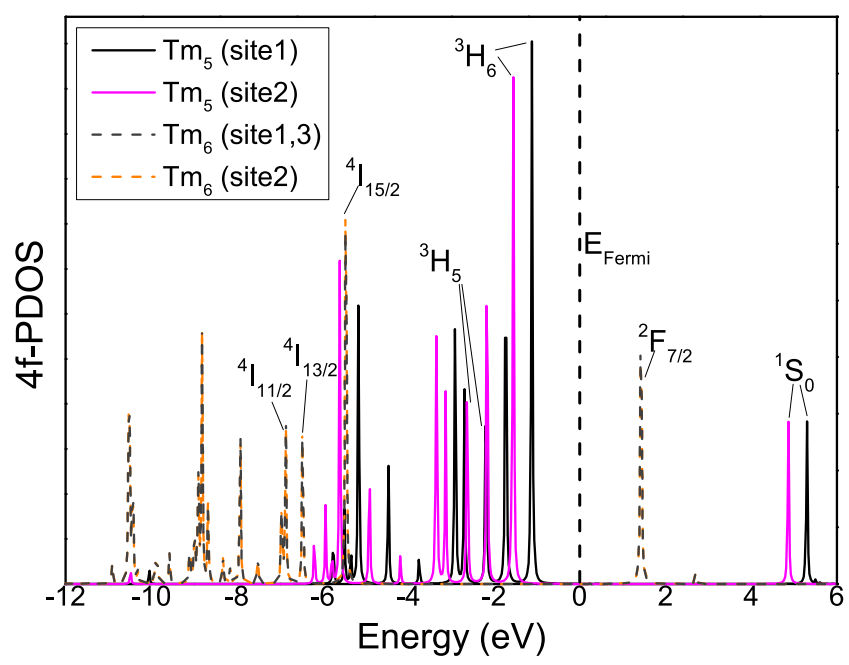

(b)

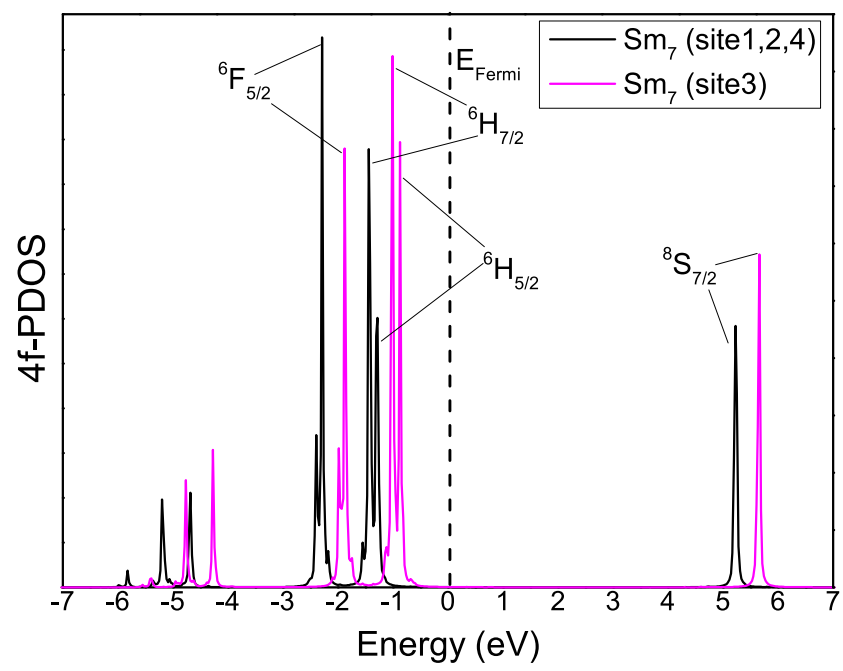

(c)

FIG. 4. (Color online) The $4 f$ PDOS calculated with the HIA is plotted for (a) trivalent $\mathrm{Tb}_{3}$, (b) divalent $\mathrm{Tm}_{5}$ and trivalent $\mathrm{Tm}_{6}$, and (c) divalent $\mathrm{Sm}_{7}$. Site 1, site 2, etc., refer to the numbered atoms in Fig. 3. the HIA. In Fig. 4, the $4 f$ PDOS is presented for (a) trivalent $\mathrm{Tb}_{3}$, (b) divalent $\mathrm{Tm}_{5}$ and trivalent $\mathrm{Tm}_{6}$ and (c) divalent $\mathrm{Sm}_{7}$. Here, the site numbers between brackets in the legend refer to the numbered atoms in Fig. 3 of the corresponding cluster size. For $\mathrm{Tb}_{3}$, all three atoms are equivalent so in Fig. 4(a) only one site is indicated. For all plots of Fig. 4, the first $4 f$ peak position below the Fermi level (corresponding to zero energy) is the same as the calculated peak positions of Fig. 3, since it is fixed to that value by the definition of the double counting [22]. Also, the atomic multiplet structure can be observed clearly in all these plots. For reader's convenience, some of the peaks are indicated. Further, from Fig. 4(b), it is clear that the valency strongly affects the spectrum, and this should, hence, provide a clear possibility to experimentally detect the predicted valences. Also the site dependence of the spectrum can be observed here and in Fig. 4(c) for $\mathrm{Sm}_{7}$.

\section{CONCLUSION AND DISCUSSION}

In this investigation, we outline how the valence of rareearth clusters evolves as a function of cluster size. From first-principles theory, combined with the Born-Haber cycle and experimental information of the atomic electronic configuration, we show that $\mathrm{Sm}$ and $\mathrm{Tb}$ clusters are respectively purely divalent and trivalent, respectively, up to a size of eight atoms. Larger clusters of $\mathrm{Tb}$ are not expected to have a valence transition, whereas from extrapolation, we predict that Sm clusters with nine-ten atoms, or more, will undergo a transition to a trivalent or a mixed valent configuration. As concerns the valence transition of $\mathrm{Tm}$, we find that there is a transition from divalent to trivalent for clusters with six atoms or more. However, the energy difference between different heterogeneous mixed valence configurations is small, as is the energy difference between such mixed valence configurations and an integer valence state. The same holds for $\mathrm{Sm}_{7}$ and in particular $\mathrm{Sm}_{8} . \mathrm{Sm}_{8}$ actually has several electronic configurations that are all within 10-100 meV. Unfortunately, the accuracy of the valence calculations used in this study is approaching these energy differences. Therefore a mixed valence situation for both Tm and Sm clusters may very well be a reality for carefully chosen cluster sizes. This holds even more true at finite temperatures, where mixed valency becomes more favorable due to its larger entropy. An experimental investigation of this prediction would be highly interesting and could potentially also shine light into the finer details of mixed valency. The decisive property of mixed valence systems $[35,36]$ is that "fast" physical measurements (with characteristic times faster than, roughly, $10^{-13} \mathrm{~s}$ ) give snapshots corresponding to a random static mixture of divalent and trivalent ions whereas "slow" (or static) measurements give the values averaged between typical for divalent and trivalent compounds. For example, the atomic volume of mixed valence compounds are intermediate between the values typical for isostructural compounds of divalent and trivalent elements. At the same time, "fast" core-level spectroscopy should give a mixture of the lines (e.g., L3 or L2 spectra) corresponding to $\mathrm{Sm}^{2+}$ and $\mathrm{Sm}^{3+}$ (or divalent and trivalent $\mathrm{Sm})$, and this seems to be the most convenient experimental way to probe the mixed valence state for the clusters. Note that the difference between homogeneous and inhomogeneous 
mixed valence, crucially important for the bulk [35,36], is not well defined for clusters, where in general not all atoms have equivalent structural positions, a fact that may be utilized to shed light on the finer details of mixed valence.

\section{ACKNOWLEDGMENTS}

We acknowledge support from the Swedish Research Council (VR), eSSENCE, STANDUPP, and the Swedish National Allocations Committee (SNIC/SNAC). The Nederlandse Organisatie voor Wetenschappelijk Onderzoek (NWO) and SURFsara are acknowledged for the usage of the LISA supercomputer and their support. The calculations were also performed on resources provided by the Swedish National Infrastructure for Computing (SNIC) at the National Supercomputer Center (NSC), the High Performance Computing Center North (HPC2N) and the Uppsala Multidisciplinary Center for Advanced Computational Science (UPPMAX). O.E. also acknowledges support from ERC (project 247062 ASD) and the KAW foundation. A.D. acknowledges financial support from KAW and KVA. M.I.K. acknowledges a support by European Research Council (ERC) Grant No. 338957. B.J. acknowledges the support from the European Research Council (ERC-2008-AdG 228074).
[1] J. Bowlan, C. N. van Dijk, A. Kirilyuk, A. Liang, S. Yin, T. Rasing, and W. A. de Heer, J. Appl. Phys. 107, 09B509 (2010).

[2] C. N. van Dijk, T. Rasing, A. Kirilyuk, J. Bowlan, A. Liang, and W. A. de Heer, J. Appl. Phys. 107, 09B526 (2010).

[3] E. Lundgren et al., Phys. Rev. Lett. 88, 136102 (2002).

[4] J. Jensen and A. R. Mackintosh, Rare Earth Magnetism (Clarendon Press, Oxford, 1991).

[5] P. Hohenberg and W. Kohn, Phys. Rev. 136, B864 (1964).

[6] W. Kohn and L. J. Sham, Phys. Rev. 140, A1133 (1965).

[7] H. Skriver, Systematics and the Properties of the Lanthanides (Reidel, Dordrecht, 1983).

[8] B. I. Min, H. J. F. Jansen, T. Oguchi, and A. J. Freeman, J. Magn. Magn. Mater. 61, 139 (1986).

[9] J. C. Duthie and D. G. Pettifor, Phys. Rev. Lett. 38, 564 (1977).

[10] L. Peters, I. Di Marco, P. Thunström, M. I. Katsnelson, A. Kirilyuk, and O. Eriksson, Phys. Rev. B 89, 205109 (2014).

[11] F. Jensen, Introduction to Computational Chemistry (Wiley, Chichester, 2006).

[12] A. Delin, L. Fast, B. Johansson, J. M. Wills, and O. Eriksson, Phys. Rev. Lett. 79, 4637 (1997).

[13] B. Johansson, Phys. Rev. B 20, 1315 (1979).

[14] For a review, see G. Kotliar, S. Y. Savrasov, K. Haule, V. S. Oudovenko, O. Parcollet, and C. A. Marianetti, Rev. Mod. Phys. 78, 865 (2006).

[15] A. I. Lichtenstein and M. I. Katsnelson, Phys. Rev. B 57, 6884 (1998).

[16] A. G. Petukhov, W. R. L. Lambrecht, and B. Segall, Phys. Rev. B 53, 4324 (1996).

[17] A. Delin, L. Fast, B. Johansson, O. Eriksson, and J. M. Wills, Phys. Rev. B 58, 4345 (1998).

[18] B. Johansson and P. Munck, J. Less-Common Met. 100, 49 (1984).

[19] W. C. Martin, R. Zalubas, and L. Hagan, NBS Natl. Stand. Ref. Data Ser. 60, 1978 (National Bureau of Standards, U. S. Department of Commerce).
[20] J. M. Wills and B. R. Cooper, Phys. Rev. B 36, 3809 (1987); also in J. M. Wills, M. Alouani, P. Andersson, A. Delin, O. Eriksson, and O. Grechnyev, Full-Potential Electronic Structure Method: Energy and Force Calculations with Density Functional and Dynamical Mean Field Theory (Springer-Verlag, Berlin, 2010).

[21] J. P. Perdew, K. Burke, and M. Ernzerhof, Phys. Rev. Lett. 77, 3865 (1996).

[22] P. Thunström, I. Di Marco, A. Grechnev, S. Lebègue, M. I. Katsnelson, A. Svane, and O. Eriksson, Phys. Rev. B 79, 165104 (2009).

[23] S. Lebègue, A. Svane, M. I. Katsnelson, A. I. Lichtenstein, and O. Eriksson, Phys. Rev. B 74, 045114 (2006).

[24] S. Lebègue, A. Svane, M. I. Katsnelson, A. I. Lichtenstein, and O. Eriksson, J. Phys. Condens. Matter 18, 6329 (2006).

[25] F. Aryasetiawan, K. Karlsson, O. Jepsen, and U. Schönberger, Phys. Rev. B 74, 125106 (2006).

[26] F. Nilsson, R. Sakuma, and F. Aryasetiawan, Phys. Rev. B 88, 125123 (2013).

[27] A. Grechnev, I. Di Marco, M. I. Katsnelson, A. I. Lichtenstein, J. Wills, and O. Eriksson, Phys. Rev. B 76, 035107 (2007).

[28] I. Di Marco, J. Minár, S. Chadov, M. I. Katsnelson, H. Ebert, and A. I. Lichtenstein, Phys. Rev. B 79, 115111 (2009).

[29] O. Grånäs, I. Di Marco, P. Thunström, L. Nordström, O. Eriksson, T. Björkman, and J. Wills, Comp. Mater. Sci. 55, 295 (2012).

[30] P. Thunström, I. Di Marco, and O. Eriksson, Phys. Rev. Lett. 109, 186401 (2012).

[31] J. A. Nelder and R. Mead, Comp. J. 7, 308 (1965).

[32] M. Domke, C. Laubschat, M. Prietsch, T. Mandel, G. Kaindl, and W. D. Schneider, Phys. Rev. Lett. 56, 1287 (1986).

[33] M. Lübcke, B. Sonntag, W. Niemann, and P. Rabe, Phys. Rev. B 34, 5184 (1986).

[34] C. Blancard et al., J. Phys. B: At. Mol. Opt. Phys. 22, L575 (1989).

[35] D. Khomskii, Sov. Phys. Uspekhi 22, 879 (1979).

[36] J. M. Lawrence, P. S. Riseborough, and R. D. Parks, Rep. Prog. Phys. 44, 1 (1981). 